apolar point-triads of a conic, the locus of points which are divided apolarly by the two triads is the conic itself and some complementary line. It remains to identify this line.

In the study of harmonic pairs the degenerate case when one of the elements is arbitrary is of great use. Then we know the other elements all coincide. Here, in the study of apolar triads, the Hessian pair of a triad and an arbitrary element are apolar with that triad. For we know that the polar pair of an arbitrary point, as to a point-triad, is harmonic with the Hessian pair.

Now, taking the triangles $t_{1}, t_{2}, t_{3}$ and $t, 0, \infty$, where $t$ is Feuerbach's point $(\S 2)$, we know, first, that lines forming an equilateral triangle determine on the line infinity a triad whose Hessian pair is the circular points $0, \infty$, so that three such lines on the one hand and an isotropic pair and an arbitrary line on the other cut the line infinity apolarly; and we know, secondly, from elementary geometry (Quar. Jour., vol. 25, p. 190) that there are two points $e$, the lines from which to $t_{1}, t_{2}, t_{3}$ form a vanishing equilateral triangle-those points, namely, which have been called the equiangular points of the triangle. Hence the line-triads from $e$ to $t_{1}, t_{2}, t_{3}$ and $t, 0, \infty$ are apolar; for the pencil is cut apolarly by the line infinity. The equiangular points do not lie on the circumcircle, hence any point on the join of the equiangular points is divided apolarly by the two triads.

Thus the complementary line is determined for this case. To pass to a covariant statement, we notice (Q.J., loc. cit.) that the line passes through the symmedian point of $t_{1} t_{2} t_{3}$, that is, through the pole of the Hessian line. Thus given two apolar triads on a conic, the points divided apolarly by them lie either on the conic itself or on the line through the poles of their Hessian lines, that is, on the line which meets the conic at the Jacobian of the Hessians of the triads.

In conclusion, it is hoped that the instance of Apolarity which has now been worked out may be useful to the student of Meyer's work, Apolarität und Rationale Curven, to which, above all, reference must be made for the projective development of the theory.

Haverford College.

\title{
AN INSTANCE WHERE A WELL-KNOWN TEST TO PROVE THE SIMPIIICITY OF A SIMPLE GROUP IS INSUFFICIEN'T.
}

IN the December number of this journal (page 64, footnote) Professor Moore asks whether an instance is known where the test used by Klein in his "Vorlesungen über das 
Ikosaeder" (1884), page 18, to prove that the icosahedrongroup of rotations is simple does not apply. The alternating substitution group of degree 68 is such an instance. The number of substitutions of the form $a b c$ is

$$
\frac{68 \cdot 67 \cdot 66}{3}=100232 \text {, }
$$

and

$$
1+100232=9.7 .37 .43 .
$$

The diophantine equation

$$
1+100232 \alpha+\ldots=d
$$

has in this case at least the following three solutions:

$$
\begin{aligned}
(\alpha, \beta, \delta, \ldots ; d)= & (1,0,0, \ldots ; 1),\left(1,1,1, \ldots ; \frac{68 !}{2}\right), \\
& (1,1,0, \ldots ; 100233) .
\end{aligned}
$$

Since every alternating group whose degree exceeds four is simply isomorphic to a number of other simple groups, this instance proves that the given test is insufficient with respect to simple groups which are not alternating. The subgroup $\Gamma_{n}$ of Professor Moore's article and its constituent groups are clearly such simple groups, if we take for $G_{n}$ the given alternating group. GEORGE A. Miller.

December 28, 1894.

\section{BRIEFER NOTICES.}

Lobachevsky Memorial Volume: 1793-1893. Celebration of the one hundredth anniversary of the birth of $N$. I. Lobachevsky. [In Russian.] Kazàn, University Press, 1894. Folio, 212 pp. With a portrait of Lobachevsky.

Is addition to a detailed account of the three days' celebration in honor of Lobachevsky, held at the University of Kazàn in November, 1893 , * this volume contains the letters and telegrams of congratulation received by the university, and some of the addresses and papers read on this occasion. Professor Suvòrov gives a somewhat popular exposition of the meaning of non-Euclidean geometry, while Professor Smirnòv discusses the same subject rather elaborately from the philosophical point of view. The other papers are historical: Mr. Iznòskov speaks of Lobachevsky's activity as a member of the Kazàn Agricultural Society; the president of the university,

\footnotetext{
* See Bulletin of the New York Mathematical Society, vol. 3, p. 201.
} 